\title{
Experimental Testing of a New Generation of Flow Diverters in Sidewall Aneurysms in Rabbits
}

Y.H. Ding, T. Tieu, and D.F. Kallmes

\begin{abstract}
BACKGROUND AND PURPOSE: The development of new generation flow-diverting devices will improve the result of flow diversion in challenging aneurysms. The Flow-Redirection Endoluminal Device system is a dual-layer flow-diversion device. The purpose of this study was to evaluate the effectiveness and safety of the Flow-Redirection Endoluminal Device in a sidewall aneurysm model and in the abdominal aorta in rabbits.
\end{abstract}

MATERIALS AND METHODS: Single Flow-Redirection Endoluminal Devices were implanted in the right common carotid artery across sidewall, vein-pouch aneurysms and within the abdominal aorta in 22 New Zealand white rabbits and followed for $1(n=5), 3(n=5), 6(n=$ 4), and 12 months $(n=8)$. Aneurysm occlusion was graded on a 3-point scale based on digital subtraction angiography (grade I, complete occlusion; grade II, near-complete occlusion; and grade III, incomplete occlusion). Toluidine blue and basic fuchsin staining was used for the evaluation of thrombus organization within the aneurysm and neck coverage with neointima. A scanning electron microscope was used for confirmation of the patency of branch vessels along with DSA.

RESULTS: Grades I and II occlusion rates were noted in 19 (86\%) and 3 (14\%) aneurysms, respectively, which indicated a 100\% rate of complete or near-complete occlusion. No parent artery and branch artery occlusion was shown on DSA. Histologic images indicated partial or complete intraluminal thrombus organization and neointima coverage across the aneurysm neck. A scanning electron microscope indicated that all the vessel branches along the length of the device remained patent.

CONCLUSIONS: The Flow-Redirection Endoluminal Device in experimental aneurysms demonstrated high rates of progressive and complete aneurysm occlusion while preserving the patency of branch vessels.

ABBREVIATIONS: FRED = Flow-Redirection Endoluminal Device; RCCA = right common carotid artery; SEM = scanning electron microscope

$F_{\text {o }}$ ow diverters have become important tools in the treatment of intracranial aneurysms as a recent disruptive technology. The Pipeline Embolization Device (Covidien, Irvine, California) is designed as a flexible microcatheter-delivered self-expanding cylindric construct composed of 48 braided strands of cobalt chromium and platinum. The Silk flow diverter (Balt Extrusion, Montmorency, France) is a retrievable device attached to a highfriction delivery system with a $200-\mathrm{cm}$ steel plunger with a $15-$ $\mathrm{mm}$-long radiopaque floppy portion that extends beyond the

Received July 10, 2014; accepted after revision September 29.

From the Neuroradiology Research Laboratory (Y.H.D., D.F.K.), Department of Radiology, Mayo Clinic, Rochester, Minnesota; and MicroVention (T.T.), Tustin, California. This work was funded by MicroVention.

Abstract previously presented at: Annual Meeting of the American Society of Neuroradiology and the Foundation of the ASNR Symposium, May 17-22, 2014; Montreal, Quebec, Canada.

Please address correspondence to Tai Tieu, MicroVention, Inc, 1311 Valencia Ave, Tustin, CA 92780; e-mail: tai.tieu@microvention.com

http://dx.doi.org/10.3174/ajnr.A4167 stent with a $45^{\circ}$ tip. The retrievability, visibility, and flexibility remain to be improved. ${ }^{1,2}$ Surpass (Stryker Neurovascular, Kalamazoo, Michigan) is a cobalt-chromium, low-porosity (metal surface area coverage, $30 \%$ ), self-expanding tubular mesh structure with high-pore attenuation. ${ }^{3}$ The high occurrence of neurologic deficits indicates that it is necessary to keep improving the performance of current flow-diverter devices. The Flow-Redirection Endoluminal Device (FRED; MicroVention, Tustin, California) was designed to increase the visibility of the device, improve its performance in patients, and reduce the occurrence of the neurologic complications.

In this study, we created a sidewall aneurysm model in rabbits and tested the FRED by combining the angiographic, histologic, and scanning electron microscope (SEM) findings at different follow-up time points.

\section{MATERIALS AND METHODS}

The FRED device is a self-expanding nickel-titanium, compliant closed-cell design compatible with a 0.027-inch Headway micro- 


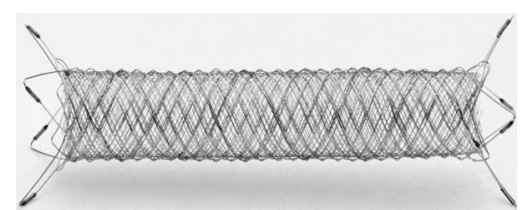

FIG 1. FRED flow-diverter system demonstrating a dual-layer design with the working section marked with a radiopaque helix to provide fluoroscopic visibility. Distal and proximal markers are evenly dispersed on its outer layer ends.

catheter (MicroVention). The FRED system has a unique duallayer design composed of a low-porosity inner flow-diverter mesh with 48 braided nitinol strands and a high-porosity outer stent with 16 nitinol struts. This dual-layer coverage is designed to put the working layer (inner part) mainly at the neck, particularly at the inflow zone of the aneurysm and to create a flow-diversion effect. The outer part, which determines the total length, serves as a protection for the inner stent. The outer stent is $3 \mathrm{~mm}$ longer than the inner flow-diverter mesh at each end so that the proximal and distal parts of the FRED can be used to cover the adjacent perforating arteries or small branches of the parent vessel with the advantage of high porosity. The FRED has 4 radiopaque markers on each end of the outer stent and 2 interwoven helical marker strands that attach to the inner and outer stents and run the entire length of the inner stent. It can be resheathed up to $85 \%$ deployment of its total length (Fig 1).

All animal procedures were performed at ISIS Services, San Carlos, California. Following approval from the Institutional Animal Care and Use Committee of ISIS, sidewall aneurysms were created in the right common carotid artery (RCCA) in $22 \mathrm{New}$ Zealand white rabbits. Details of the creation procedure have been published previously. ${ }^{4}$ Briefly, anesthesia was induced by intravenous administration of xylazine $(1 \mathrm{mg} / \mathrm{kg})$ and ketamine $(10 \mathrm{mg} /$ $\mathrm{kg}$ ) and was maintained with isoflurane $(2 \%-3 \%)$ in oxygen $(2$ $\mathrm{L} / \mathrm{min}$ ). Using a sterile technique, we made an 8 -cm paramedian incision in the neck. The RCCA and right external jugular vein were exposed and dissected. After permanent ligation of the proximal and distal sides of the right external jugular vein, a 1-cm vein pouch was harvested between the 2 sides (right external jugular vein proximal and distal). A 5-mm arteriotomy of the RCCA was made, and ligation was performed to temporarily occlude the proximal and distal sides of the arteriotomy. Heparin (150 U/kg) was injected intravenously through the ear vein before anastomosis. An end-to-side anastomosis between the vein pouch and the RCCA by using an 8-0 Prolene suture (Ethicon, Cincinnati, Ohio) was performed.

Three weeks after creation, patency of all the aneurysms and parent arteries was confirmed by DSA before FRED deployment. The detailed procedure has been published before. ${ }^{5,6}$ Briefly, a 5F sheath was advanced on 1 side of the femoral artery via cutdown, followed by a $5 \mathrm{~F}$ Chaperon guiding catheter (MicroVention). A Headway 27 microcatheter (MicroVention) was advanced into the distal end of parent artery (RCCA) over a Traxcess 14EX microguidewire (MicroVention) through the guide catheter. The first FRED ( $3.5 \mathrm{~mm}$ outer stent diameter $\times 17 / 11 \mathrm{~mm}$ ) was deployed across the aneurysm neck within the RCCA. The second device ( $4.0 \mathrm{~mm}$ outer stent diameter $\times 18 / 12 \mathrm{~mm}$ ) was deployed within the abdominal aorta crossing multiple lumbar arteries. DSA was performed through the guide catheter immediately after deployment. Aspirin $(10 \mathrm{mg} / \mathrm{kg})$ and clopidogrel $(10 \mathrm{mg} / \mathrm{kg}$ ) were given daily 2 days before implantation and were continued until 90 days after treatment.

Follow-up DSA was performed at 1 month $(n=5), 3$ months $(n=5), 6$ months $(n=4)$, and 12 months $(n=8)$ after treatment. Animals were sacrificed with a lethal injection of pentobarbital at each time point after DSA follow-up. The RCCA, including the aneurysm sac and abdominal aorta with lumbar arteries, was immediately fixed in 10\% neutral buffered formalin.

Degrees of intra-aneurysmal flow disruption immediately after device deployment and before sacrifice were graded on a 3-point scale based on DSA images, including grade I (complete flow cessation, no flow within the aneurysm), grade II (near-complete flow, $<10 \%$ residual flow), and grade III (incomplete occlusion, $\geq 10 \%$ residual flow). ${ }^{7}$ The degree of aneurysm occlusion at follow-up was compared with the immediate postembolization images by using another 3-point scale, including stable aneurysm occlusion, progressive aneurysm occlusion, and aneurysm recanalization. Patency of parent artery and lumbar artery branches was also evaluated.

Carotid aneurysm samples were dehydrated in a graded series of ethanol and embedded in methyl methacrylate plastic. After polymerization of the plastic, 2- to 3-mm transverse sections were sawed from the proximal and distal ends of the FREDs and from the area of the aneurysm neck. The sections were ground to a thickness of $42-50 \mu \mathrm{m}$ by using Linear Grinding technology (Exakt Technologies, Oklahoma City, Oklahoma), polished, and stained with toluidine blue and basic fuchsin. All sections containing the aneurysm sac and aneurysm neck were evaluated by light microscopy to score histologic changes on the basis of specific parameters. The tissues within the aneurysm were categorized as unorganized or organized connective tissue. The degree of endothelialized neointima across the neck was also assessed.

DSA and a scanning electron microscope were used to evaluate the patency of the lumbar arterial branch ostia on the aortic luminal surface. Before processing, the samples were opened longitudinally to expose the luminal surface and the FRED implants were photographed. The sample was rinsed in $0.1-\mathrm{mol} / \mathrm{L}$ sodium phosphate buffer and then postfixed in $1 \%$ osmium tetroxide for 30 minutes. The samples were then dehydrated in a graded series of ethanol. After critical point drying, the tissue was mounted and sputter coated with gold; the sample was visualized by using a scanning electron microscope (magnification range X15-X600; Hitachi, Tokyo, Japan). Tissue processing and the histopathology report (including SEM results) were offered by CVpath Institute, Gaithersburg, Maryland.

Histologic sections containing the proximal, mid-, and distal sections were analyzed with a National Institute of Standards and Technology calibrated microscope system (IP Lab software, Rockville, Maryland) under a BX51 light microscope (Olympus, Melville, New York). The average value of each histologic factor at the above 3 locations was calculated. The cross-sectional areas (internal elastic lamina [IEL] and lumen) of each stented section were measured. The percentage of luminal narrowing was calculated with the following formula:

$\%$ Stenosis $=[1-($ Lumen Area/IEL Area $)] \times 100$.

AJNR Am J Neuroradiol 36:732-36 Apr 2015 www.ajnr.org 
The extent of stenosis was classified as 4 categories: minimal $(<20 \%)$, mild (20\%-35\%), moderate ( $>35 \%$ to $<50 \%)$, and marked ( $\geq 50 \%$ ).

Neointima formation and endothelial coverage was semiquantified and expressed as the percentage of the lumen circumference covered by neointima and endothelium, respectively. Their different extents were categorized as the following: minimal ( $<25 \%$ of the luminal surface, score: 1 ), mild $(25 \%-50 \%$ of the luminal surface, score: 2$)$, moderate $(51 \%-75 \%$ of the luminal surface, score: 3 ), and marked ( $>75 \%$ of the luminal surface, score: 4$)$.

\section{RESULTS}

Mean aneurysm sizes (including the aneurysm neck, width, and height) of the 22 aneurysms are shown in Table 1 . There was no significant difference in aneurysm neck, width, and height among groups at different time points $(P>.05)$.

Five rabbits were sacrificed at 1 month after FRED deployment: Four aneurysms demonstrated incomplete occlusion immediately after device deployment and 1 aneurysm showed near-

Table 1: Mean aneurysm sizes at different time points

\begin{tabular}{lccc}
\hline Time & Neck $(\mathbf{m m})$ & Width $(\mathbf{m m})$ & Height $(\mathbf{m m})$ \\
\hline 1 Mo & $4.1 \pm 1.1$ & $6.5 \pm 2.1$ & $7.0 \pm 1.7$ \\
$3 \mathrm{Mo}$ & $3.5 \pm 1.0$ & $5.5 \pm 1.6$ & $5.5 \pm 1.2$ \\
$6 \mathrm{Mo}$ & $3.7 \pm 1.1$ & $6.3 \pm 1.7$ & $5.9 \pm 1.4$ \\
$12 \mathrm{Mo}$ & $3.6 \pm 1.0$ & $5.5 \pm 1.5$ & $6.0 \pm 1.4$ \\
\hline
\end{tabular}

complete occlusion. One month after treatment, 4 (4/5, 80\%) aneurysms (including the aneurysm shown as having near-complete occlusion immediately after treatment) were completely occluded and 1 aneurysm showed near-complete occlusion (Fig 2A-C).

In the next follow-up group, 4 aneurysms were incompletely occluded immediately after device deployment, and 1 aneurysm showed near-complete occlusion. Three months after treatment, complete occlusion was shown in $4(4 / 5,80 \%)$ aneurysms (including the aneurysm shown as having near-complete occlusion immediately after treatment) and near-complete occlusion was indicated in 1 aneurysm.

Four aneurysms at the 6-month point indicated incomplete occlusion immediately after device deployment. Six months posttreatment, all $(4 / 4,100 \%)$ aneurysms were completely occluded.

Eight aneurysms were included at the 12-month point: Seven aneurysms were incompletely occluded immediately after device deployment and 1 aneurysm showed near-complete occlusion. Twelve months after treatment, 7 (7/8, 88\%) aneurysms (including the aneurysm shown as having near-complete occlusion immediately after treatment) were completely occluded, while 1 aneurysm showed near-complete occlusion (Fig 3A-C).

In total, $19(86 \%)$ of 22 aneurysms were completely occluded (grade I) during follow-up and 3 (14\%) of 22 aneurysms showed near-complete occlusion (grade II). Thus $100 \%$ of the aneurysms indicated complete or near-complete occlusion. All aneurysms

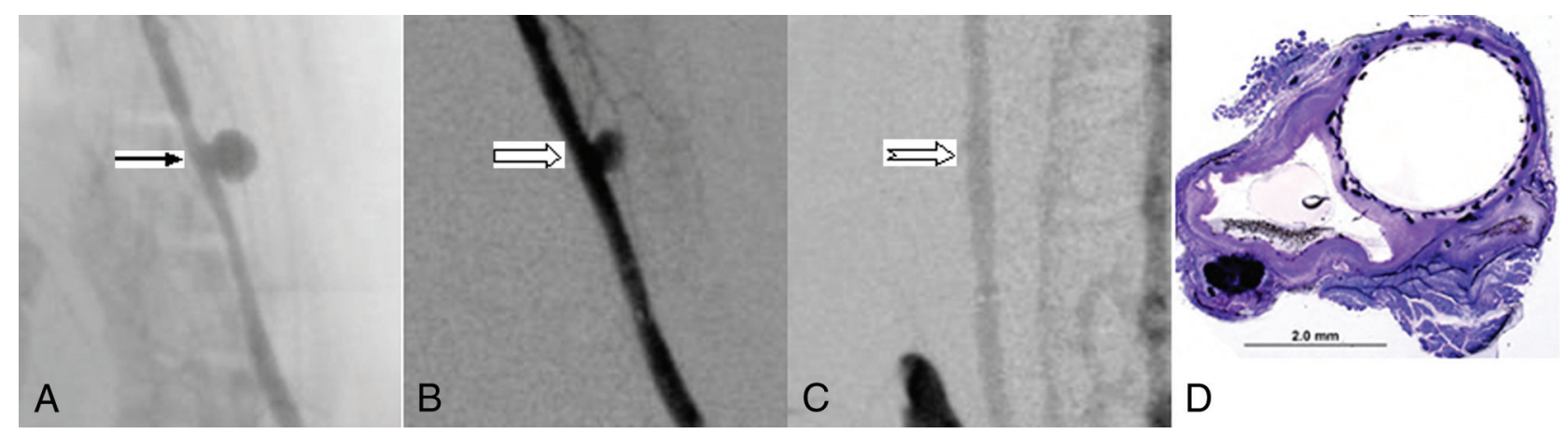

FIG 2. A, Anteroposterior digital subtraction angiogram shows the aneurysm (arrow) before treatment. B, DSA image immediately after device deployment shows aneurysm partial occlusion (block arrow). C, DSA image at 1 month shows complete aneurysm occlusion (notched arrow). D, Photomicrograph stained with toluidine blue and basic fuchsin shows an empty aneurysm sac completely sealed with neointimal growth incorporating the FRED struts and mesh spanning the neck, magnification $15 \times$.

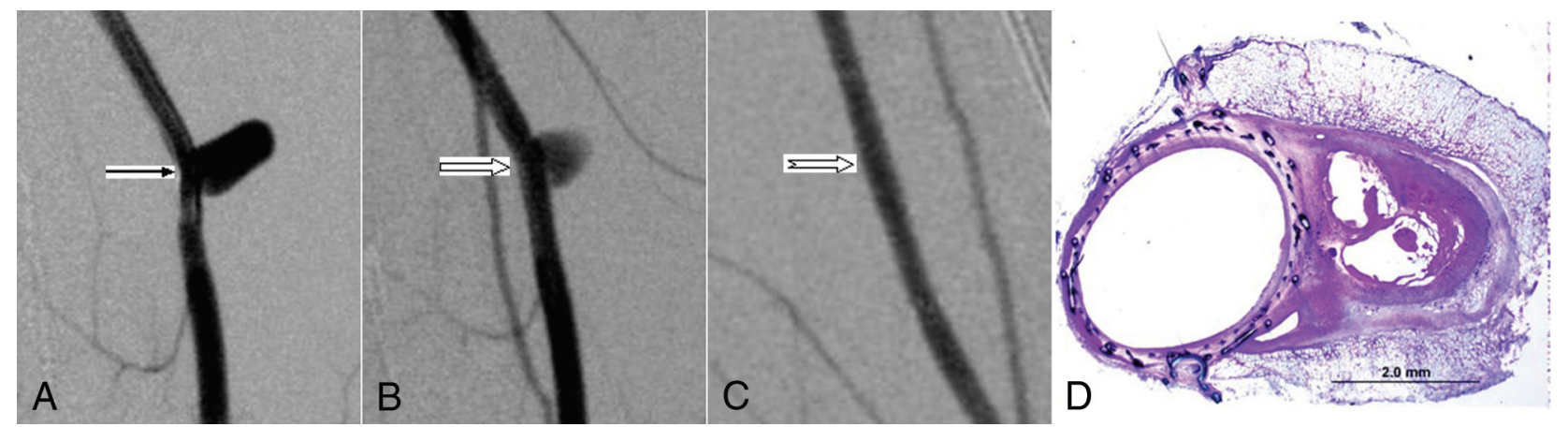

FIG 3. $A$, Anteroposterior digital subtraction angiogram shows the aneurysm (arrow) before treatment. B, DSA image immediately after device deployment shows partial aneurysm occlusion (block arrow). C, DSA image at 12 months shows complete aneurysm occlusion (notched arrow). $D$, Photomicrograph stained with toluidine blue and basic fuchsin shows partially organized thrombus within the aneurysm sealed with complete neointima incorporating the FRED struts and mesh across the neck, magnification $15 \times$. 
showed progressive occlusion when follow-up imaging was compared with imaging immediately after treatment (Table 2). Parent arteries remained patent in all cases.

Partially or completely organized thrombus, unorganized fibrin, and an empty aneurysm cavity were shown at each time point. Neointimal growth incorporated the device and completely excluded the aneurysm neck in 19 aneurysms, which included 2 of the 3 incompletely occluded aneurysms shown on DSA. Incomplete neointima across the neck was indicated in 3 aneurysms, of which 2 were completely occluded as shown on DSA (Figs $2 D$ and $3 D$ ).

Nineteen $(86 \%)$ cases showed marked neointima formation and endothelial coverage. Two cases ( 1 in the 3-month group and 1 in 12-month group) had both mild neointima formation and endothelial coverage. One case in the 12-month group showed moderate neointima formation and mild endothelial coverage. The average stenosis percentage in each group was $<30 \%$, which indicated only mild intima hyperplasia (Table 3 ).

All lumbar arteries remained patent in 4 groups during the follow-up period; the patency was confirmed by DSA before sacrifice and with an SEM (Fig 4).

Table 2: Aneurysm occlusion summary

\begin{tabular}{lcccccc}
\hline & \multicolumn{2}{c}{$\begin{array}{c}\text { Immediately after } \\
\text { Treatment }\end{array}$} & & \multicolumn{2}{c}{ Before Sacrifice } \\
\cline { 2 - 3 } \cline { 5 - 6 } & Incomplete & $\begin{array}{c}\text { Near- } \\
\text { Complete }\end{array}$ & & Complete & $\begin{array}{c}\text { Near- } \\
\text { Complete }\end{array}$ \\
\hline 1-Mo group $(n=5)$ & 4 & 1 & & 4 & 1 \\
3-Mo group $(n=5)$ & 4 & 1 & & 4 & 1 \\
6-Mo group $(n=4)$ & 4 & 0 & & 4 & 0 \\
12-Mo group $(n=8)$ & 7 & 1 & & 7 & 1 \\
\hline
\end{tabular}

Table 3: Histologic data at different time points

\begin{tabular}{lccc}
\hline & $\begin{array}{c}\text { Neointima } \\
\text { Formation }\end{array}$ & $\begin{array}{c}\text { Endothelial } \\
\text { Coverage }\end{array}$ & $\begin{array}{c}\text { Stenosis } \\
\text { Percentage }\end{array}$ \\
\hline 1-Mo group & $4 \pm 0$ & $4 \pm 0$ & $28.7 \pm 7.0$ \\
3-Mo group & $3.6 \pm 0.9$ & $3.6 \pm 0.9$ & $22.9 \pm 3.9$ \\
6-Mo group & $4 \pm 0$ & $4 \pm 0$ & $20.0 \pm 6.0$ \\
12-Mo group & $3.6 \pm 0.7$ & $3.5 \pm 0.9$ & $23.2 \pm 4.0$ \\
\hline
\end{tabular}

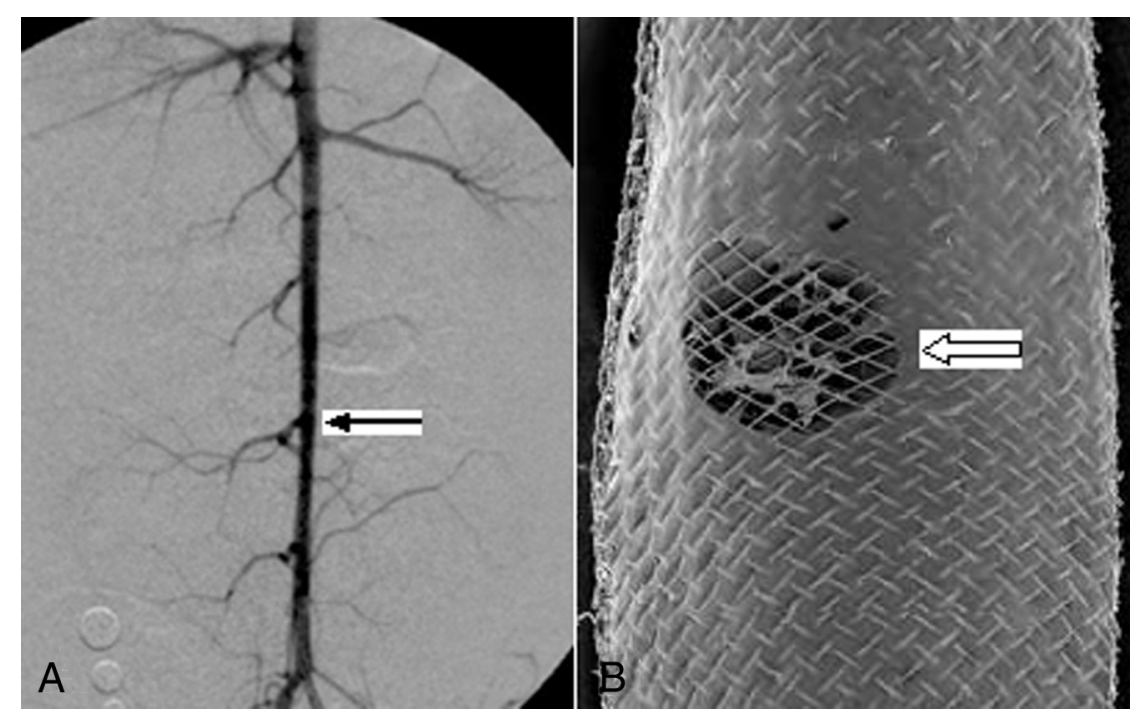

FIG 4. A, DSA 12 months after device deployment across the lumbar arteries (arrow), indicating patent lumbar arteries. $B$, SEM shows the open orifice of the lumbar artery (block arrow), magnification 15X.

\section{DISCUSSION}

In this study, we tested the FRED in sidewall aneurysms in rabbits. A high rate of complete aneurysm occlusion was achieved up to 12 months following implantation. Histologic findings showed that aneurysms were filled with unorganized or organized thrombus with the neck sealed by neointimal incorporation within the FRED struts and meshes. In a vein patch sidewall aneurysm model, the device demonstrated high rates of progressive and complete aneurysm occlusion while also preserving the patency of small arteries arising from the abdominal aorta.

Using a rabbit elastase-induced aneurysm model in rabbits, our group tested the performance of the Pipeline and reported the angiographic and histologic features at different periods after deployment. The aneurysm lumen was filled with unorganized, partial, or completely organized thrombus. Endothelial cells were found to cover the device struts across the aneurysm neck, and they were contiguous with the endothelium of the parent artery or aorta. Parent arteries and small, adjacent branch vessels remained patent; the patency was also confirmed by a scanning electric microscope. The rate of complete or near-complete aneurysm occlusion were $88 \%,{ }^{5}$ which was lower than that in this study (100\%) by using a surgical sidewall aneurysm model. In addition, moderately thick intimal hyperplasia was noted at the distal stent portion of the parent artery in the elastase-induced aneurysm model, which is mainly because the devices were relatively oversized in the distal aspect of the parent artery. In the sidewall surgical aneurysm model, because the proximal and distal sides of parent artery have similar sizes, no obvious difference of neointimal hyperplasia in the proximal and distal parent artery was shown. ${ }^{5}$

Other researchers reported their initial clinical experience with the FRED, and the preliminary results are encouraging. In 33 patients with 37 aneurysms with up to 12 months' followup, the complete aneurysm occlusion rate was $80 \%(4 / 5)$ at 4-6 months and $100 \%(8 / 8)$ at 7-12 months after FRED deployment. Both the mortality and permanent morbidity rates were $0 \% .{ }^{8}$ A high aneurysm complete occlusion rate $(7 / 8,88 \%)$ was achieved at 3-month follow-up in another report. ${ }^{9}$

This study had several limitations. We included only a small number of subjects at each time point. In addition, although there were no significant differences of aneurysm sizes (neck, width, and height) among different groups, aneurysm shapes were not similar in some cases; these shapes might explain the difference in healing in various aneurysms.

\section{CONCLUSIONS}

In a vein patch sidewall aneurysm model, the FRED demonstrated high rates of progressive and complete aneurysm occlusion with minimal parent artery stenosis and preservation of small arterial branches arising from the abdominal aorta. 


\section{ACKNOWLEDGMENTS}

The authors thank ISIS Services, San Carlos, California, led by the Chief Scientific Officer/Founder Jorge Garcia for professional management from Amy Valera, Project Manager; and technical support from Victor Avalos, Surgical Specialist; Benigno Mills, Surgical Specialist; Adam Loo, operating room technician; and Eric Chu, operating room technician, for the laboratory animal testing.

Disclosures: Tai Tieu—RELATED: MicroVention, Comments: I am a full-time employee of MicroVention Inc; thus, I receive a salary from MicroVention. My employment with MicroVention is not dependent on any publication. I receive a salary from MicroVention regardless of whether this work is published; UNRELATED: Patents (planned, pending, or issued): MicroVention, Comments: All patents are the right of MicroVention. For pending or issued patents, I receive less than $\$ 1000$ from MicroVention. David F. Kallmes—RELATED: Grant: MicroVention, ${ }^{\star}$ Comments: support for research personnel; Support for Travel to Meetings for the Study or Other Purposes: MicroVention, ${ }^{*}$ Comments: travel to lab for device implant; UNRELATED: Board Membership: GE Healthcare (Cost-Effectiveness Board); Consultancy: ev3,* Medtronic,* Comments: planning and implementation of clinical trials; Grants/ Grants Pending: MicroVention, ${ }^{*}$ Codman, ${ }^{*}$ Surmodics, ${ }^{*}$ Sequent, ${ }^{\star}$ ev $3,{ }^{*}$ Comments: preclinical and clinical research; Royalties: University of Virginia Patent Foundation (Spine Fusion). *Money paid to the institution.

\section{REFERENCES}

1. Tan LA, Keigher KM, Munich SA, et al. Thromboembolic complications with Pipeline embolization device placement: impact of pro- cedure time, number of stents and pre-procedure $\mathrm{P} 2 \mathrm{Y} 12$ reaction unit (PRU) value. J Neurointerv Surg 2015;7:217-21

2. Cirillo L, Leonardi M, Dall'olio M, et al. Complications in the treatment of intracranial aneurysms with Silk stents: an analysis of $\mathbf{3 0}$ consecutive patients. Interv Neuroradiol 2012;18:413-25

3. De Vries JD, Boogaarts J, Norden AV, et al. New generation of flow diverter (Surpass) for unruptured intracranial aneurysms: a prospective single-center study in 37 patients. Stroke 2013;44:1567-77

4. Ding YH, Tieu T, Kallmes DF. Creation of sidewall aneurysm in rabbits: aneurysm patency and growth follow-up. J Neurointerv Surg 2014;6:29-31

5. Kallmes DF, Ding YH, Dai D, et al. A new endoluminal, flowdisrupting device for treatment of saccular aneurysms. Stroke 2007;38:2346-52

6. Kallmes DF, Ding YH, Dai D, et al. A second-generation, endoluminal, flow-disrupting device for treatment of saccular aneurysms. AJNR Am J Neuroradiol 2009;30:1153-58

7. Brinjikji W, Lanzino G, Cloft HJ, et al. Patency of the posterior communicating artery after flow diversion treatment of internal carotid artery aneurysms. Clin Neurol Neurosurg 2014;120:84-88

8. Kocer N, Islak C, Kizilkilic O, et al. Flow Re-direction Endoluminal Device in treatment of cerebral aneurysms: initial experience with short-term follow-up results. J Neurosurg 2014;120:1158-71

9. Poncyljusz W, Sagan L, Safranow K, et al. Initial experience with implantation of novel dual layer flow-diverter device FRED. Wideochir Inne Tech Malo Inwazyjne 2013;8:258-64 